Journal of Advanced Research in Fluid Mechanics and Thermal Sciences

Journal homepage: www.akademiabaru.com/arfmts.html ISSN: 2289-7879

\title{
Caputo Fractional MHD Casson Fluid Flow Over an Oscillating Plate with Thermal Radiation
}

\author{
Ridhwan Reyaz ${ }^{1}$, Yeou Jiann Lim ${ }^{1}$, Ahmad Qushairi Mohamad ${ }^{1, *}$, Muhammad Saqib ${ }^{1}$, Sharidan \\ Shafie $^{1}$
}

1 Department of Mathematical Sciences, Fakulti Sains, Universiti Teknologi Malaysia, Skudai, 81310 Johor Bahru, Johor, Malaysia

\begin{tabular}{|c|c|}
\hline ARTICLE INFO & ABSTRACT \\
\hline $\begin{array}{l}\text { Keywords: } \\
\text { Casson fluid; Caputo derivative; Laplace } \\
\text { transform; Thermal radiation }\end{array}$ & $\begin{array}{l}\text { The effect of the thermal radiation on the MHD Casson fluid along with the fractional } \\
\text { derivative in an oscillating vertical plate is elucidated. More exactly, the Caputo } \\
\text { fractional model is utilized in developing the governing equations. Besides, the } \\
\text { influence of the buoyancy force due to the temperature gradient has also been } \\
\text { considered. The derived fractional partial differential equations are converted into } \\
\text { ordinary differential equations by using the Laplace transform technique and then are } \\
\text { solved for analytical solutions via the characteristic method. The inversion of the } \\
\text { Laplace transformation is obtained through the numerical approach of Zakian. The } \\
\text { effects of various physical parameters on the velocity and temperature profiles, Nusselt } \\
\text { number, and skin friction have been analyzed and depicted in graphs and tables. The } \\
\text { distribution of the velocity and temperature either in viscous or Casson fluid do } \\
\text { enhance by the fractional parameter. }\end{array}$ \\
\hline
\end{tabular}

\section{Introduction}

Non-newtonian fluids have been recognized as one of the most popular research topics due to the wide applications in production engineering, chemical engineering as well as the manufacturing process. A non-Newtonian fluid is described as fluids that do not act according to Newton's law of viscosity. Newton's law of viscosity states that the viscosity of fluid remains the same despite the various amount of stress acting on it. The non-Newtonian fluid behaves such that it will be less viscous or sticky when more pressure is applied. Corn starch fluid is an example of a non-Newtonian fluid. Recent research on non-Newtonian fluid can be found in [1-3]. Due to the complexity of the nonNewtonian fluids, the classical Navier-Stokes theory does not express the fluid adequately, and also no single equation as of today could describe the non-Newtonian fluids comprehensively. As such, several models have been developed over the years to investigate the properties of these fluids i.e., Maxwell model and Casson Model [4]. One of the most popular subclasses of non-Newtonian fluid is the Casson fluid, a model that exhibits the yield stress impact of the fluid. Casson fluid behaves such

\footnotetext{
* Corresponding author.

E-mail address: ahmadqushairi@utm.my
}

https://doi.org/10.37934/arfmts.85.2.145158 
that it will act like a solid when the shear stress applied to it is less than its yield stress and it will be in motion when shear stress applied is more than its yield stress [5]. The Casson fluid model was first introduced by Casson [6] in 1959 whilst investigating the rheological data of pigment ink in a printer. Examples of recent research on Casson fluid are [7-9]. Over the past few decades, research on Casson fluid has increased drastically. Frigaard [10] did an extensive review on simple stress yield fluids, this includes Casson fluid. Meanwhile, Gbadeyan et al., [11] provided a review on the effects of magnetohydrodynamic (MHD) and thermal radiation on Casson fluid. Other than that, Kumar and Pai [12] did an extensive study on a Casson fluid passing though a porous circular bearing. Yusof et al., [13] on the other hand studied the effects of radiation on Casson fluid passing through an exponentially accelerated riga plate with permeability and slip effect in mind. In the hopes further investigating and peeling the theoretical and mathematical models on Casson fluid, researchers are visiting the idea of fractional derivates an it's impact on fluid flow models.

Fractional derivatives are simply defined as derivatives with orders between 0 and 1 . It was first mentioned by L'Hospital when he wrote a letter to Leibniez [14] asking what would happen if the $n$ notation on a derivative would be a complex or fractional number. Since then, many researchers has tried their hands on defining fractional derivative. This includes Riemann, Abel and Liouville [15]. The Riemann-Liouville, Grunwald-Letnikov and Caputo are a few of the most popular definitions used to date [16-17]. Research on fractional derivatives has skyrocketed over the past few decades. Fractional derivatives have been applied in many fields including mechanical engineering, civil engineering, numerical analysis, etc. For example, Cao et al., [18] used Riemann-Liouville fractional derivative to calculate the shear stress of a beam by performing Laplace transform on the fractional Euler-Lagrange equation. Meanwhile, Gómez-aguilar et al., [19] applied not only the RiemannLiouville but as well as the Grfinwald-Letnikov, Liouville-Caputo, and Caputo-Fabrizio fractional derivative to model electrical circuits and analyze them. Jamil et al., [20] did an investigation on the effects of magnetism of a blood flow within an inclined cylindrical tube while incorporating the Caputo-Fabrizio fractional derivative. More applications of the fractional derivative are shown in [21] and [22]. The Caputo derivative, developed by Italian physicist Michele Caputo in 1967, is used to model an occurrence that considers past interactions and nonlocal problems [23]. A recent publication on Caputo derivative in the field of fluid mechanics is done by Yang et al., [24] where the properties of heat and flow transfer of fractional Maxwell fluid above the different thickness of stretching sheet was discussed. The article concluded that stronger elastic characteristics are exhibited when a larger fractional parameter is used. Other recent publications on the Caputo derivative includes [25-27].

By considering a free convection flow of Casson fluid in a porous medium, Khalid et al., [28] obtained the exact solutions using Laplace transform. Khan et al., [29] extended the study by introducing fractional calculus to the generalised Casson fluid and considering oscillating boundary conditions. Again, the solution was obtained through the Laplace transform. Later, Ali et al., [30] acknowledged a fractional generalised Casson fluid with an infinite plate instead of a finite plate. Saqib et al., [31] have deliberated the Casson fluid flows under the influence of the fractional parameter in the presence of first-order chemical reactions and considering the slip effect.

In all the above-mentioned literature, no attempt has been given on investigating the effect of the noninteger derivatives, free convection, and thermal radiation on the flow of the MHD Casson fluid that is driven by a vertical oscillating infinite plate. Therefore, the present study aims to extend the work of Ali et al., [30] by introducing thermal radiation and the MHD effect in the Casson fluid flow and heat transfer along with an oscillating plate. The Caputo fractional derivative model is applied to deriving the governing equations. Laplace transformation is practiced to transform the nonlinear governing partial differential equations into ordinary differential equations. Analytical 
solutions of the ODEs are then determined by using the undetermined coefficient method and Laplace's inversion of the solutions will be obtained through Zakian's method. Effects of the fractional parameter, Prandtl number, thermal radiation, time, Grashof number, magnetic parameter as well as plate oscillation frequency on the fluid flow and heat analysis have been discussed and plotted graphically via Mathcad 15 software.

\section{Problem Definition}

An unsteady incompressible MHD Casson fluid over an infinite oscillating vertical flat plate is considered. The cartesian coordinate system is applied in the present study and $y$ is taken in the direction normal to the plate. The flow is constricted to $y>0$. Initially at $t=0$, the plate is at rest, and the plate and the ambient fluid are assumed to have a constant temperature $T_{\infty}$. As time progresses, $t>0$, the plate begins to oscillate with velocity $\frac{A}{t} \sin (\omega t)$, where $A$ is the plate's acceleration, while $\omega$ is the frequency of the plate's oscillations. Meanwhile, the temperature of the plate is radiatively increased to $T_{W}$ and remained constant thereafter. The radiation effect is also considered such that the heat flux is applied perpendicular to the plate. The temperature and velocity is dependent on variable $y$ and $t$. By utilising the Boussinesq's approximation and considering a unidirectional flow, the acquired momentum and energy equations are as follows [32-34]

$$
\begin{aligned}
& \frac{\partial u(y, t)}{\partial t}=\frac{\mu}{\rho}\left(1+\frac{1}{\beta}\right) \frac{\partial^{2} u(y, t)}{\partial y^{2}}+g \beta_{T}\left(T-T_{\infty}\right)-\frac{1}{\rho} \sigma B_{0}^{2} u(y, t), \\
& \frac{\partial T}{\partial t}=k \frac{1}{\rho C_{p}} \frac{\partial^{2} T}{\partial y^{2}}-\frac{\partial q_{r}}{\partial y}
\end{aligned}
$$

with initial and boundary conditions

$$
\begin{array}{ccrl}
u(y, 0) & =0, & T(y, 0) & =T_{\infty}, \\
u(0, t)=\frac{A}{t} \sin (\omega t), & T(0, t) & =T_{w}, \\
u(\infty, t) & \rightarrow 0, & T(\infty, t) & \rightarrow T_{\infty},
\end{array}
$$

where $u$ is the velocity and $T$ is the temperature of the fluid, $\beta$ is the parameter for Casson fluid, $\mu$ is viscosity, $g$ is gravitational acceleration, $\beta_{T}$ is the thermal expansion coefficient, $B_{0}$ is the magnetic parameter, $C_{P}$ is the specific heat under constant pressure and $k$ is thermal conductivity.

Using Rosseland's approximation,

$$
q_{r}=\frac{4 \sigma^{*}}{3 k^{*}} \frac{\partial T^{4}}{\partial y},
$$

the thermal radiation term, $\frac{\partial q_{r}}{\partial y}$ can be obtained by differentiating Equation (4) with respect to y. where $\sigma^{*}$ is the Stefan-Boltzman constant and $k^{*}$ is the mean absorption coefficient. Using the following non-dimensional variables in Eq. (5), the governing equations and the corresponding boundary conditions, 
$u^{*}=\frac{u}{A} t, \quad y^{*}=\frac{A}{v t} y$,
$t^{*}=\frac{A^{2}}{t v}, \quad T^{*}=\frac{T-T_{\infty}}{T_{W}-T_{\infty}}, \quad \omega^{*}=\frac{v t^{2}}{A^{2}} \omega$.

and removing the asterisk notation, the final non-dimensional governing equations from Eq. (1) and (2) take the form such as [35-38]

$\frac{\partial u(y, t)}{\partial t}=\frac{1}{\beta_{0}} \frac{\partial^{2} u(y, t)}{\partial y^{2}}-M^{2} u(y, t)+G r T(y, t)$

$\frac{\partial T(y, t)}{\partial t}=\left(1+\frac{4}{3} N\right) \frac{1}{\operatorname{Pr}} \frac{\partial^{2} T(y, t)}{\partial y^{2}}$,

with initial and boundary conditions

$$
\begin{gathered}
u(y, 0)=0, \quad T(y, 0)=0, \\
u(0, t)=\sin (\omega t), \quad T(0, t)=1, \\
u(\infty, t) \rightarrow 0, \quad T(\infty, t) \rightarrow 0,
\end{gathered}
$$

where

$$
\begin{aligned}
& \frac{1}{\beta_{0}}=1+\frac{1}{\beta}, \quad M^{2}=\frac{\sigma B_{0}^{2}}{\rho} \frac{v}{A^{2}} t^{2}, \quad G r=\frac{v g \beta_{T}\left(T_{W}-T_{\infty}\right)}{A^{3}} t^{3}, \\
& N=\frac{4 \sigma^{*} T_{\infty}^{3}}{k^{*} k}, \quad \operatorname{Pr}=\frac{\mu C_{P}}{k},
\end{aligned}
$$

and $\beta_{0}, M^{2}, G r, N$, Pr are dimensionless Casson parameter, the magnetic parameter, Grashof number, thermal radiation parameter, and Prandtl number respectively.

To investigate the impact of fractional derivative on the velocity and temperature profiles of the Casson fluid, Eq. (6) and (7) are modified to the fractional governing equations such as

$$
\begin{aligned}
& D_{t}^{\alpha} u(y, t)=\frac{1}{\beta_{0}} \frac{\partial^{2} u(y, t)}{\partial y^{2}}-M^{2} u(y, t)+\operatorname{Gr} T(y, t), \\
& D_{t}^{\alpha} T(y, t)=\left(1+\frac{4}{3} N\right) \frac{1}{\operatorname{Pr}} \frac{\partial^{2} T(y, t)}{\partial y^{2}},
\end{aligned}
$$

where

$$
D_{t}^{\alpha} f(x, t)=\frac{1}{\Gamma(1-\alpha)} \int_{0}^{t} \frac{f^{\prime}(y, t)}{(t-\mu)^{\alpha}} d \mu
$$

is the Caputo fractional derivative with order $\alpha$ and $\Gamma(\cdot)$ and is Bernoulli's gamma function. 


\subsection{Problem Solution}

To investigate the behavior of the fluid, an analytical solution via Laplace transform and numerical inversion Laplace transform by Zakin method are constructed.

Eq. (8), (10) and (11) is first transformed into an ordinary system of equations via Laplace transform such as

$$
\begin{aligned}
& \frac{d^{2} \bar{u}(y, q)}{d \bar{y}^{2}}-\beta_{0}\left(q^{\alpha}-M^{2}\right) \bar{u}(y, q)=-G r \beta_{0} \bar{T}(y, q), \\
& \frac{d^{2} \bar{T}(y, q)}{d \bar{y}^{2}}-\frac{q^{\alpha} P r}{1+\frac{4}{3} N} \bar{T}(y, q)=0,
\end{aligned}
$$

and boundary conditions

$$
\begin{array}{cl}
\bar{u}(y, 0)=0, & \bar{T}(y, 0)=0, \\
\bar{u}(0, q)=\frac{\omega}{q^{2}+\omega^{2}}, & \bar{T}(0, q)=\frac{1}{q}, \\
\bar{u}(\infty, q) \rightarrow 0, & \bar{T}(\infty, q) \rightarrow 0 .
\end{array}
$$

where the bar notation symbolises Laplace notations. Solving Eq. (13) and (14) subjected to boundary conditions (15) yields

$$
\begin{aligned}
\bar{u}(y, q)= & \frac{\omega}{q^{2}+\omega^{2}} \exp \left(-y \sqrt{\beta_{0}\left(q^{\alpha}-M^{2}\right)}\right)-\frac{D_{1}}{q\left(q^{\alpha}+D_{2}\right)} \exp \left(-y \sqrt{\beta_{0}\left(q^{\alpha}-M^{2}\right)}\right) \\
& +\frac{D_{1}}{q\left(q^{\alpha}+D_{2}\right)} \exp \left(-y \sqrt{q^{\alpha} \operatorname{Pr}_{0}}\right), \\
\bar{T}(y, q)= & \frac{1}{q} \exp \left(-y \sqrt{q^{\alpha} \operatorname{Pr}_{0}}\right),
\end{aligned}
$$

where

$$
D_{1}=\frac{-G r \beta_{0}}{P r_{0}-\beta_{0}}, \quad D_{2}=\frac{\beta_{0} M^{2}}{P r_{0}-\beta_{0}}, \quad \operatorname{Pr}_{0}=\frac{P r}{1+\frac{4}{3} N}
$$

To recover the final solution for the velocity and temperature profiles, Zakian's method as stated in the Eq. (18) for computing the numerical solutions of the inverse Laplace transform is employed [39, 40].

$$
f(t)=\frac{2}{t} \sum_{i=1}^{n} \operatorname{Re}\left\{K_{i} F\left(\frac{\alpha_{i}}{t}\right)\right\} .
$$




\subsubsection{Skin friction coefficient and Nusselt number}

Skin friction coefficient and Nusselt number for Casson fluid in non-dimensional form is given as

$$
\begin{aligned}
& C_{f}=\left.\frac{1}{\beta_{0}} \frac{\partial u(y, t)}{\partial y}\right|_{y=0}, \\
& N u=\left.\frac{\partial T(y, t)}{\partial y}\right|_{y=0} .
\end{aligned}
$$

\section{Result and Discussions}

The characteristic of the Caputo fractional MHD Casson fluid over an oscillating plate under the effects of thermal radiation is investigated through observations of velocity and temperature profiles that have been computed by the analytical solutions. The numerical inversion solutions of Eq. (16) and (17) are derived by utilizing Zakian's method as defined in the Eq. (18) and the results are plotted using Mathcad 15.

It is observed from Figure 1, the Prandtl number has decreased the Casson fluid velocity. This is due to the decrement in buoyancy force. Meanwhile, Figure 2 exhibits the behavior of fluid with increment in thermal radiation parameter. The increment of the thermal radiation value has escalated the stored kinetic energy in the fluid, thus the fluid's motion is enhanced. On the other hand, as time increases, the fluid motion is intensified as depicted in Figure 3. Figure 4 illustrates that the distribution of the fluid is an increasing function of the Casson parameter. When the Casson parameter approaches infinity, the fluid behaves more like a Newtonian fluid result in lower viscosity and a higher fluid velocity. Next, Figure 5 has exhibited the response of the fluid velocity due to the increment of the Grashof number. The fluid moves faster when the magnitude of the Gr is raised. Grashof number is defined as the ratio of the buoyancy force to the viscous force that acting on a fluid and fluid motion is linearly dependent on the buoyancy force. Thus, increasing the Grashof value would simultaneously escalate the buoyant force and de-escalate the viscous force that acting on the fluid, consequently increases the fluid motion. Figure 6 displays fluid velocity for different values of the magnetic parameter. It is observed that with an increment of the magnetic parameter, fluid velocity would decrease. This is mostly due to the Lorentz force generated from the magnetic field which has slowed down the fluid motion. Figure 7 demonstrates that a higher velocity profile is produced by a large frequency of the oscillating plate.

Figure 8 presents the fluid's temperature profile with different values of Prandtl number. An increment of Prandtl value results in a decrease in fluid temperature. As the Prandtl number is the ratio of momentum diffusivity and thermal diffusivity, therefore, the fluid temperature is inversely proportional to the Prandtl number as observed in Figure 8. In contrast, the fluid temperature increases with the increment of the thermal radiation parameter. As the thermal radiation parameter grows, external heat is applied to the fluid and hence the fluid temperature is enhanced. This behavior can be observed in Figure 9. Further, Figure 10 has shown that the fluid temperature would also be improved by a greater time. Noteworthy, escalating the fractional parameter, would increase the fluid's velocity and temperature as seen in Figure 1 to Figure 10. Other than that, fluid would also reach a steady-state faster when the fractional parameter is decreased. 


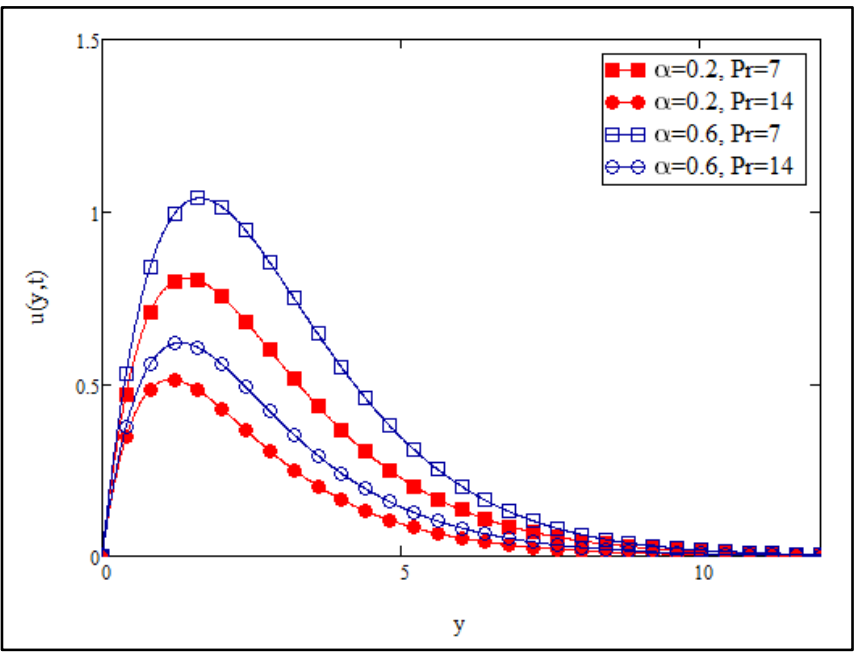

Fig 1. Velocity profile for different values of $\alpha$ and $\operatorname{Pr}$

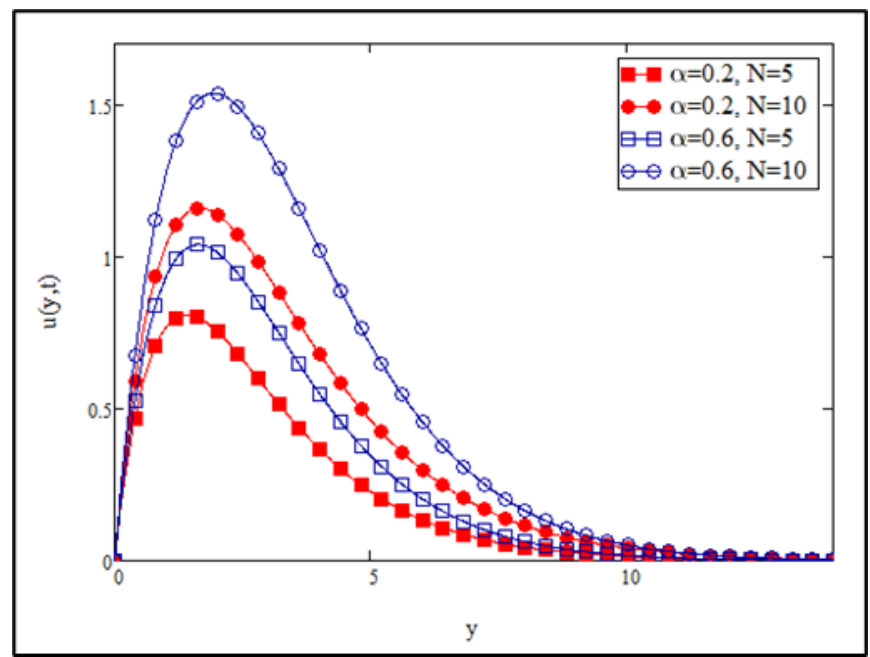

Fig. 2. Velocity profile for different values of $\alpha$ and $N$

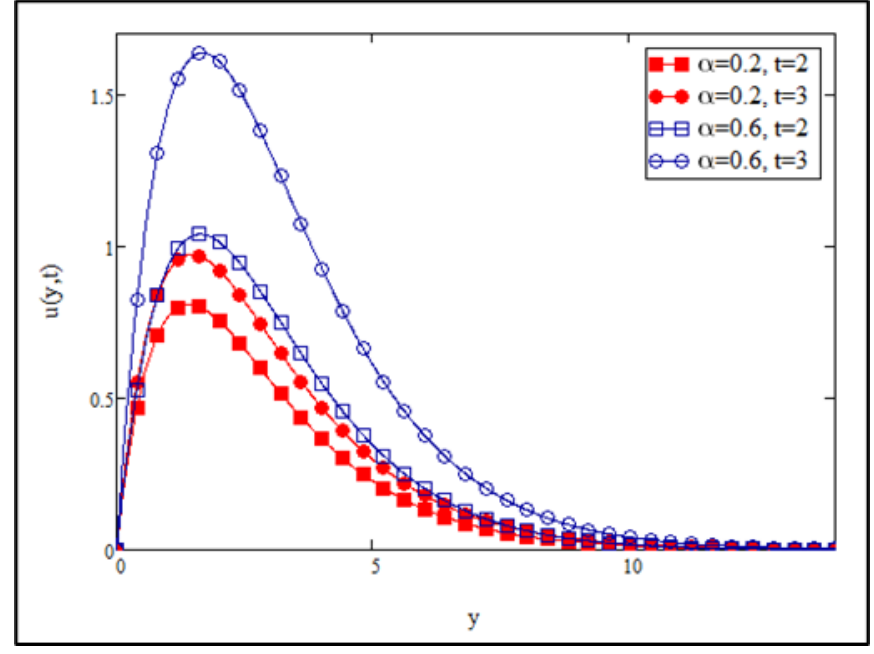

Fig. 3. Velocity profile for different values of $\alpha$ and $t$ 


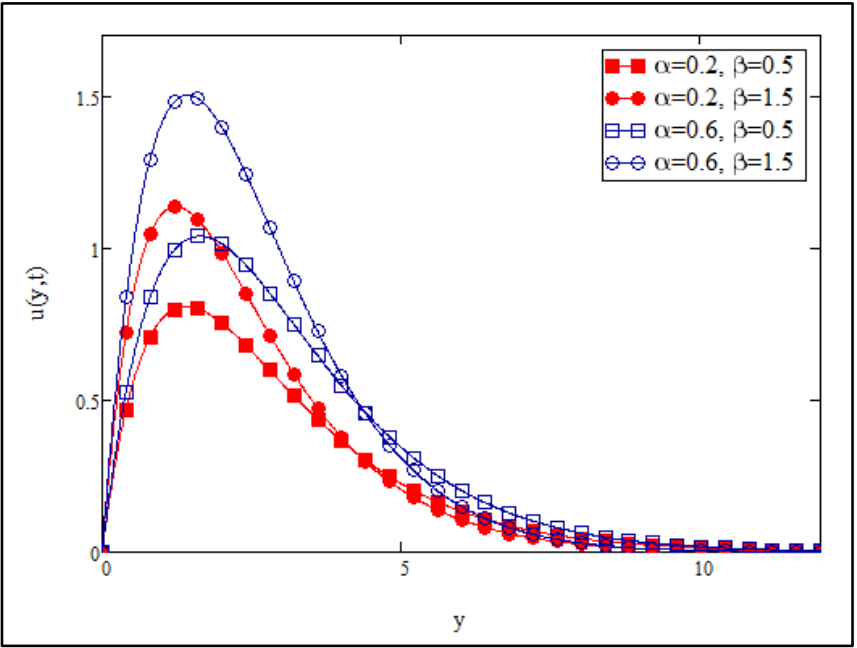

Fig. 4. Velocity profile for different values of $\alpha$ and $\beta$

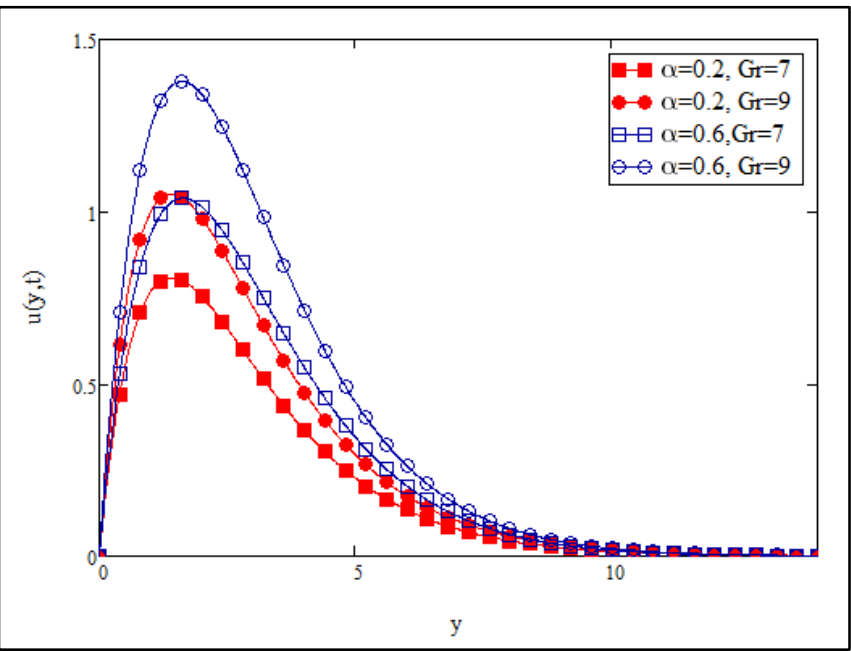

Fig.5. Velocity profile for different values of $\alpha$ and $G r$

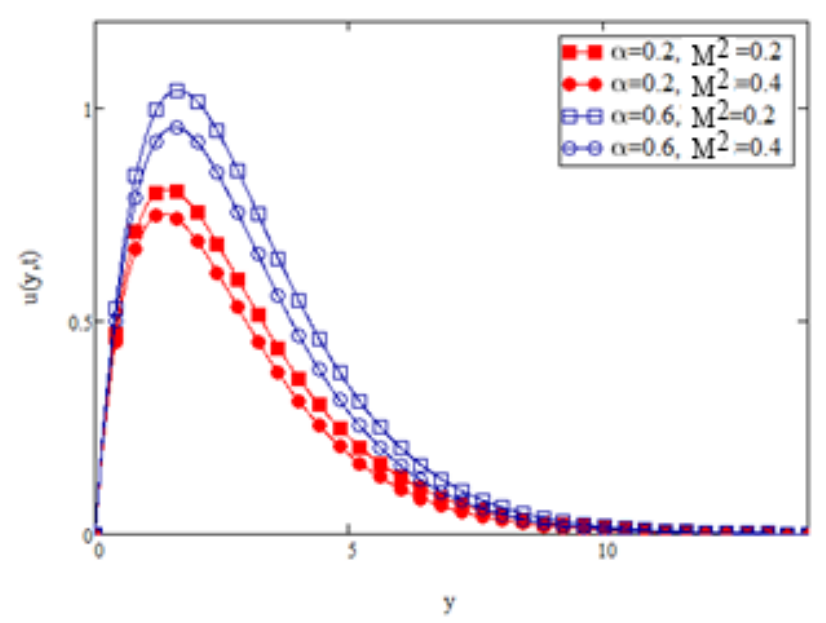

Fig. 6. Velocity profile for different values of $\alpha$ and $M^{2}$ 


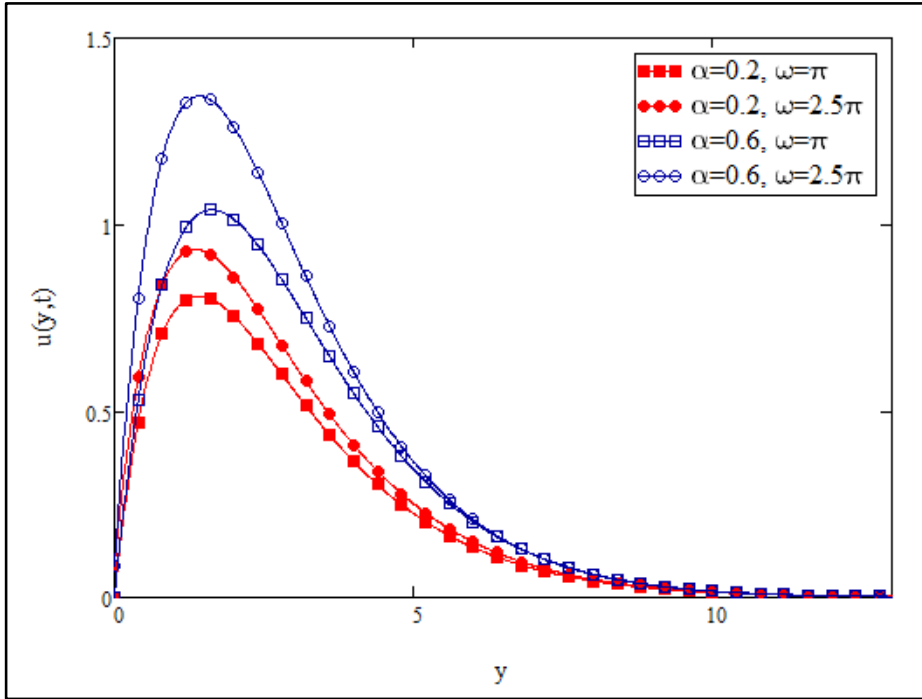

Fig. 7. Velocity profile for different values of $\alpha$ and $\omega$

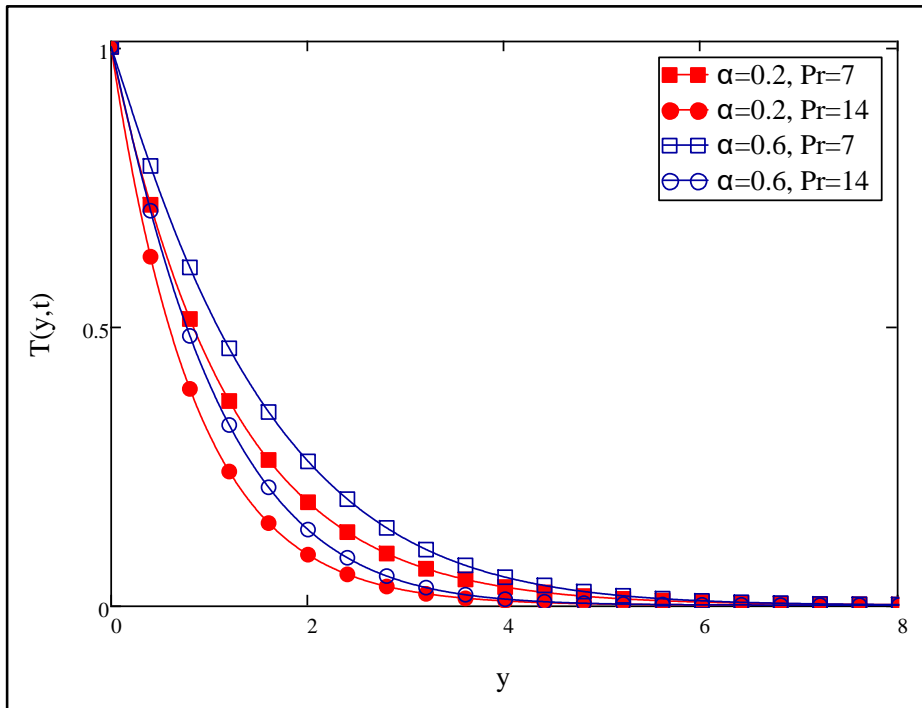

Fig. 8. Temperature profile for different values of $\alpha$ and $\operatorname{Pr}$

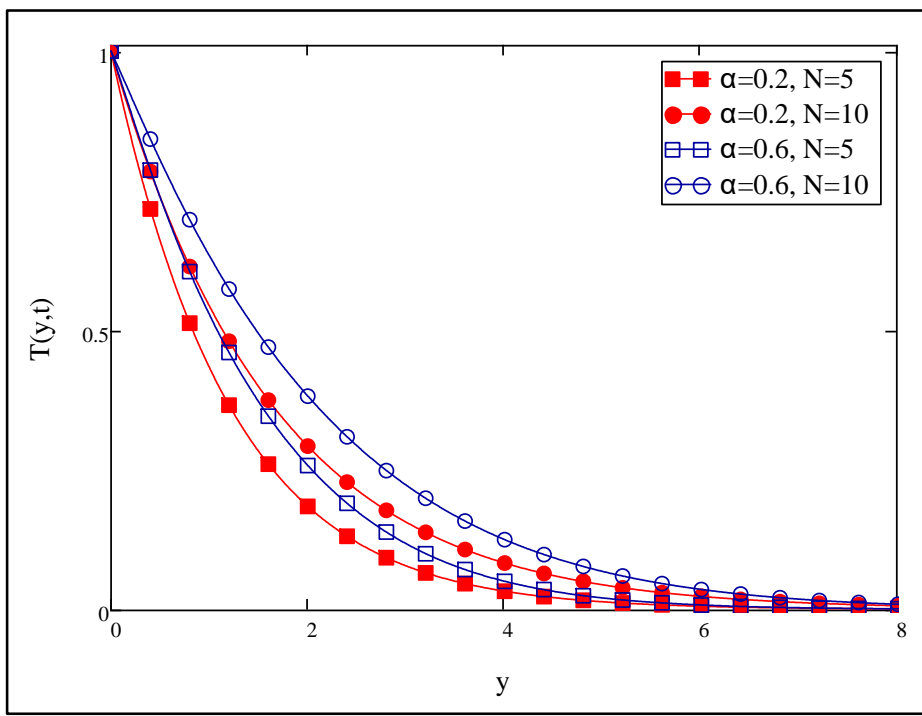

Fig. 9. Temperature profile for different values of $\alpha$ and $N$ 


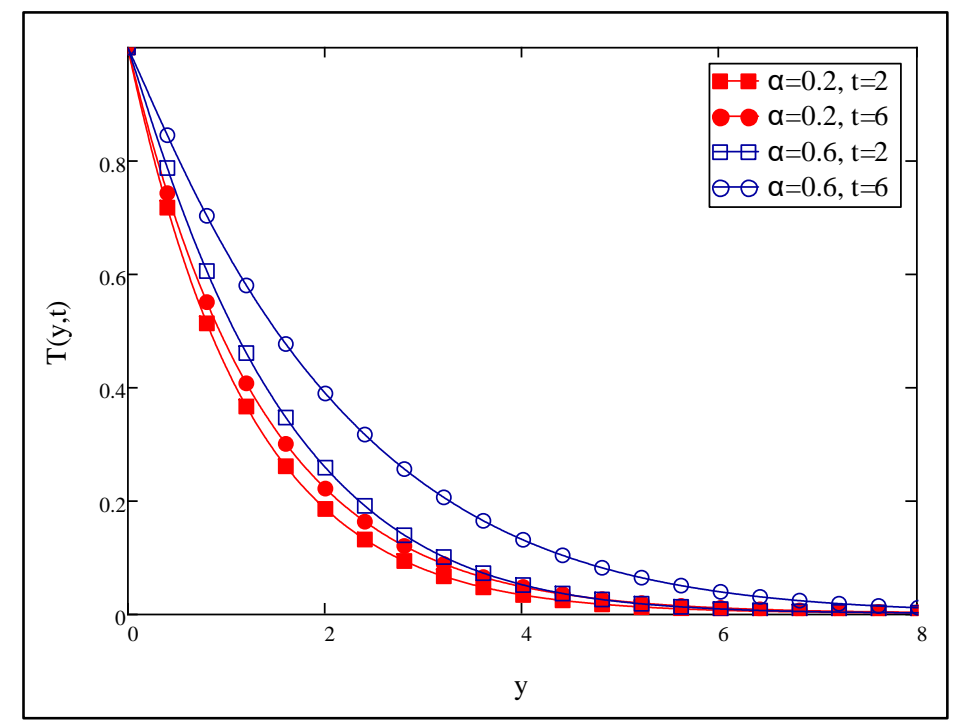

Fig. 10. Temperature profile for different values of $\alpha$ and $t$

Table 1 has displayed a summary of fluid behavior with increment in different values of the embedded parameters. In Table 2, the skin friction coefficients for different interested parameters values are tabulated. It is observed that the skin friction coefficient would be amplified if values of $\alpha$ , $N, t, \beta, G r$, and $\omega$ are elevated. In contrast, the coefficient would be lowered by increasing the values of $\operatorname{Pr}$ and $M^{2}$. Meanwhile, the Nusselt number with different parametric values has been portrayed in Table 3. The Nusselt number tends to increase with increasing parametric values of $\alpha$, $N, t$ and decrease with increments in $\operatorname{Pr}$. The Nusselt number is defined as the ratio of convection heat transfer and fluid conduction heat transfer, a high Nusselt number value would most definitely increase the fluid's temperature as the heat is conducted at a higher rate as seen in Figure 8 to Figure 10.

\section{Table 1}

Behaviour of fluid with different parametric values

\begin{tabular}{lll}
\hline Increment of parametric variable & Velocity profile & Temperature profile \\
\hline$\alpha$ & $\uparrow$ & $\uparrow$ \\
$\operatorname{Pr}$ & $\downarrow$ & $\downarrow$ \\
$N$ & $\uparrow$ & $\uparrow$ \\
$t$ & $\uparrow$ & $\uparrow$ \\
$\beta$ & $\uparrow$ & - \\
$G r$ & $\uparrow$ & - \\
$M^{2}$ & $\downarrow$ & - \\
$\omega$ & $\uparrow$ & - \\
\hline
\end{tabular}


Table 2

Skin friction coefficient variation

\begin{tabular}{llllllllll}
\hline$\alpha$ & $\operatorname{Pr}$ & $N$ & $t$ & $\beta$ & $G r$ & $M^{2}$ & $\omega$ & $C_{f}$ & Profile \\
\hline 0.2 & 7 & 5 & 2 & 0.5 & 7 & 0.2 & $\pi$ & 0.52 & - \\
$\mathbf{0 . 6}$ & 7 & 5 & 2 & 0.5 & 7 & 0.2 & $\pi$ & 0.55 & $\uparrow$ \\
0.2 & 14 & 5 & 2 & 0.5 & 7 & 0.2 & $\pi$ & 0.411 & $\downarrow$ \\
0.2 & 7 & 10 & 2 & 0.5 & 7 & 0.2 & $\pi$ & 0.625 & $\uparrow$ \\
0.2 & 7 & 5 & $\mathbf{3}$ & 0.5 & 7 & 0.2 & $\pi$ & 0.603 & $\uparrow$ \\
0.2 & 7 & 5 & 2 & $\mathbf{1 . 5}$ & 7 & 0.2 & $\pi$ & 1.494 & $\uparrow$ \\
0.2 & 7 & 5 & 2 & 0.5 & 14 & 0.2 & $\pi$ & 1.069 & $\uparrow$ \\
0.2 & 7 & 5 & 2 & 0.5 & 7 & $\mathbf{0 . 6}$ & $\pi$ & 0.486 & $\downarrow$ \\
0.2 & 7 & 5 & 2 & 0.5 & 7 & 0.2 & $\mathbf{2 . 5}$ & 0.562 & $\uparrow$ \\
\hline
\end{tabular}

Table 3

Nusselt number variation

\begin{tabular}{llllll}
\hline$\alpha$ & $\operatorname{Pr}$ & $N$ & $t$ & $N u$ & Profile \\
\hline 0.2 & 7 & 5 & 2 & -0.826 & - \\
$\mathbf{0 . 6}$ & 7 & 5 & 2 & -0.534 & $\uparrow$ \\
0.2 & $\mathbf{1 4}$ & 5 & 2 & -1.169 & $\downarrow$ \\
0.2 & 7 & $\mathbf{1 0}$ & 2 & -0.604 & $\uparrow$ \\
0.2 & 7 & 5 & $\mathbf{3}$ & -0.793 & $\uparrow$ \\
\hline
\end{tabular}

\section{Conclusion}

This paper aims to investigate the free convection flow of MHD Casson fluid over an oscillating plate with the effects of thermal radiation. Caputo fractional derivative is concerned in the present study. From the finding, we can conclude

i. The velocity of fluid would increase if values of $\alpha, N, t, \beta, G r$, and $\omega$ is increased. In contrast, the velocity decreases if values of $\operatorname{Pr}$ and $M^{2}$ were to increase.

ii. The temperature of fluid would increase if values of $\alpha, N$, and $t$ is increased. In contrast, the temperature decreases if values of $\mathrm{Pr}$ is increased.

iii. An increment in fractional parameter, $\alpha$ would increase both fluid velocity and temperature and would result in a long time for the fluid to reach a steady state.

iv. Skin friction complements fluid velocity. An increase in $\alpha, N, t, \beta, G r$, and $\omega$ would increase the skin friction and an increase in $\operatorname{Pr}$ and $M^{2}$ would decrease the skin friction.

v. Nusselt number complements fluid temperature. An increase in $\alpha, N$, and $t$ would increase Nusselt number and an increase in Pr would decrease the Nusselt number.

\section{Acknowledgment}

The authors would like to acknowledge the Ministry of Higher Education Malaysia and Research Management Centre-UTM, Universiti Teknologi Malaysia (UTM) for financial support through vote numbers FRGS/1/2018/STG06/UTM/02/4, 08G33 and 17J98. 


\section{References}

[1] Afrouzi, Hamid Hassanzadeh, Majid Ahmadian, Abouzar Moshfegh, Davood Toghraie, and Ashkan Javadzadegan. "Statistical analysis of pulsating non-Newtonian flow in a corrugated channel using Lattice-Boltzmann method." Physica A: Statistical Mechanics and its Applications $535 \quad$ (2019): 122486. https://doi.org/10.1016/j.physa.2019.122486

[2] Mehmood, Obaid Ullah, Ayesha Aleem Qureshi, Humaira Yasmin, and Salah Uddin. "Thermo-mechanical analysis of non Newtonian peristaltic mechanism: Modified heat flux model." Physica A: Statistical Mechanics and its Applications 550 (2020): 124014. https://doi.org/10.1016/j.physa.2019.124014

[3] Mohebbi, Rasul, Amin Amiri Delouei, Amin Jamali, Mohsen Izadi, and Abdulmajeed A. Mohamad. "Pore-scale simulation of non-Newtonian power-law fluid flow and forced convection in partially porous media: thermal lattice Boltzmann method." Physica A: Statistical Mechanics and Its Applications 525 (2019): 642-656. https://doi.org/10.1016/i.physa.2019.03.039

[4] Pramanik, S. "Casson fluid flow and heat transfer past an exponentially porous stretching surface in presence of thermal radiation." Ain Shams Engineering Journal 5, no. $1 \quad$ (2014): $205-212$. https://doi.org/10.1016/i.asej.2013.05.003

[5] Sheikh, Mariam, and Zaheer Abbas. "Homogeneous-heterogeneous reactions in stagnation point flow of Casson fluid due to a stretching/shrinking sheet with uniform suction and slip effects." Ain Shams Engineering Journal 8 , no. 3 (2017): 467-474. https://doi.org/10.1016/j.asej.2015.09.010

[6] Casson, N. "A flow equation for pigment-oil suspensions of the printing ink type." Rheology of disperse systems (1959).

[7] Naqvi, Syed Muhammad Raza Shah, Taseer Muhammad, and Mir Asma. "Hydromagnetic flow of Casson nanofluid over a porous stretching cylinder with Newtonian heat and mass conditions." Physica A: Statistical Mechanics and its Applications 550 (2020): 123988. https://doi.org/10.1016/j.physa.2019.123988

[8] Liu, Chunyan, Liancun Zheng, Ping Lin, Mingyang Pan, and Fawang Liu. "Anomalous diffusion in rotating Casson fluid through a porous medium." Physica A: Statistical Mechanics and its Applications 528 (2019): 121431. https://doi.org/10.1016/j.physa.2019.121431

[9] Maiti, S., S. Shaw, and G. C. Shit. "Caputo-Fabrizio fractional order model on MHD blood flow with heat and mass transfer through a porous vessel in the presence of thermal radiation." Physica A: Statistical Mechanics and its Applications 540 (2020): 123149. https://doi.org/10.1016/i.physa.2019.123149

[10] Frigaard, lan. "Simple yield stress fluids." Current Opinion in Colloid \& Interface Science 43 (2019): 80-93. https://doi.org/10.1016/i.cocis.2019.03.002

[11] Gbadeyan, J. A., E. O. Titiloye, and A. T. Adeosun. "Effect of variable thermal conductivity and viscosity on Casson nanofluid flow with convective heating and velocity slip." Heliyon 6, no. 1 (2020): e03076. https://doi.org/10.1016/i.heliyon.2019.e03076

[12] Kumar, Sampath, and Nithyanand Pai. "Flow of casson fluid through circular porous bearing." CFD Letters 12, no. 7 (2020): 48-56. https://doi.org/10.37934/cfdl.12.7.4856

[13] Yusof, Nur Syamila, Siti Khuzaimah Soid, Mohd Rijal Illias, Ahmad Sukri Abd Aziz, and Nor Ain Azeany Mohd Nasir. "Radiative Boundary Layer Flow of Casson Fluid Over an Exponentially Permeable Slippery Riga Plate with Viscous Dissipation." Journal of Advanced Research in Applied Sciences and Engineering Technology 21, no. 1 (2020): 41-51. https://doi.org/10.37934/araset.21.1.4151

[14] Leibniz, Gottfried Wilhelm. "Letter to John Frederick, Duke of Brunswick-Hanover." In Philosophical Papers and Letters, pp. 259-262. Springer, Dordrecht, 1989.

[15] Ross, Bertram, ed. Fractional calculus and its applications: proceedings of the international conference held at the University of New Haven, June 1974. Vol. 457. Springer, 2006. https://doi.org/10.1007/BFb0067096

[16] Malinowska, Agnieszka, and Delfim Torres. "Fractional calculus of variations for a combined Caputo derivative." Fractional Calculus and Applied Analysis 14, no. 4 (2011): 523-537. https://doi.org/10.2478/s13540$\underline{011-0032-6}$

[17] Loverro, Adam. "Fractional calculus: history, definitions and applications for the engineer." Rapport technique, Univeristy of Notre Dame: Department of Aerospace and Mechanical Engineering (2004): 1-28.

[18] Labora, Daniel Cao, António M. Lopes, and JA Tenreiro Machado. "Time-fractional dependence of the shear force in some beam type problems with negative Young modulus." Applied Mathematical Modelling 80 (2020): 668-682. https://doi.org/10.1016/i.apm.2019.11.054

[19] Gómez-Aguilar, J. F., H. Yépez-Martínez, R. F. Escobar-Jiménez, C. M. Astorga-Zaragoza, and J. Reyes-Reyes. "Analytical and numerical solutions of electrical circuits described by fractional derivatives." Applied Mathematical Modelling 40, no. 21-22 (2016): 9079-9094. https://doi.org/10.1016/j.apm.2016.05.041 
[20] Jamil, Dzuliana Fatin, Salah Uddin, M. Ghazali Kamardan, and Rozaini Roslan. "The Effects of Magnetic Blood Flow in an Inclined Cylindrical Tube Using Caputo-Fabrizio Fractional Derivatives." CFD Letters 12, no. 1 (2020): 111-122.

[21] Berdyshev, A. S., A. Cabada, and B. Kh Turmetov. "On solvability of some boundary value problem for polyharmonic equation with boundary operator of a fractional order." Applied Mathematical Modelling 39, no. 15 (2015): 45484569. https://doi.org/10.1016/i.apm.2015.01.006

[22] Li, Changpin, and Hengfei Ding. "Higher order finite difference method for the reaction and anomalous-diffusion equation." Applied Mathematical Modelling 38, no. $\quad$ 15-16 $\quad$ (2014): $3802-3821$. https://doi.org/10.1016/i.apm.2013.12.002

[23] Atangana, Abdon. Fractional operators with constant and variable order with application to geo-hydrology. Academic Press, 2017.

[24] Yang, Weidong, Xuehui Chen, Xinru Zhang, Liancun Zheng, and Fawang Liu. "Flow and heat transfer of double fractional Maxwell fluids over a stretching sheet with variable thickness." Applied Mathematical Modelling 80 (2020): 204-216. https://doi.org/10.1016/i.apm.2019.11.017

[25] Wu, Guo-Cheng, and Dumitru Baleanu. "Variational iteration method for the Burgers' flow with fractional derivatives-new Lagrange multipliers." Applied Mathematical Modelling 37, no. 9 (2013): 6183-6190. https://doi.org/10.1016/i.apm.2012.12.018

[26] Yuttanan, Boonrod, and Mohsen Razzaghi. "Legendre wavelets approach for numerical solutions of distributed order fractional differential equations." Applied Mathematical Modelling 70 (2019): $350-364$. https://doi.org/10.1016/j.apm.2019.01.013

[27] Pan, Mingyang, Liancun Zheng, Fawang Liu, Chunyan Liu, and Xuehui Chen. "A spatial-fractional thermal transport model for nanofluid in porous media." Applied Mathematical Modelling 53 (2018): 622-634. https://doi.org/10.1016/j.apm.2017.08.026

[28] Khalid, Asma, Lim Yeou Jiann, Ilyas Khan, and Sharidan Shafie. "Exact solutions for unsteady free convection flow of carbon nanotubes over an oscillating vertical plate." In AIP Conference Proceedings, vol. 1830, no. 1, p. 020054. AIP Publishing LLC, 2017. https://doi.org/10.1063/1.4980917

[29] Khan, Ilyas, Nehad Ali Shah, and Dumitru Vieru. "Unsteady flow of generalized Casson fluid with fractional derivative due to an infinite plate." The European Physical Journal Plus 131, no. 6 (2016): 1-12. https://doi.org/10.1140/epjp/i2016-16181-8

[30] Ali, Farhad, Nadeem Ahmad Sheikh, Ilyas Khan, and Muhammad Saqib. "Solutions with Wright function for time fractional free convection flow of Casson fluid." Arabian Journal for Science and Engineering 42, no. 6 (2017): 25652572. https://doi.org/10.1007/s13369-017-2521-3

[31] Saqib, Muhammad, Farhad Ali, Ilyas Khan, and Nadeem Ahmad Sheikh. "Heat and mass transfer phenomena in the flow of Casson fluid over an infinite oscillating plate in the presence of first-order chemical reaction and slip effect." Neural Computing and Applications 30, no. 7 (2018): 2159-2172. https://doi.org/10.1007/s00521-016$\underline{2810-x}$

[32] Khan, Arshad, Dolat Khan, Ilyas Khan, Farhad Ali, Faizan ul Karim, and Muhammad Imran. "MHD flow of sodium alginate-based Casson type nanofluid passing through a porous medium with Newtonian heating." Scientific reports 8, no. 1 (2018): 1-12. https://doi.org/10.1038/s41598-018-26994-1

[33] Sheikh, Nadeem Ahmad, Farhad Ali, Muhammad Saqib, Ilyas Khan, Syed Aftab Alam Jan, Ali Saleh Alshomrani, and Metib Said Alghamdi. "Comparison and analysis of the Atangana-Baleanu and Caputo-Fabrizio fractional derivatives for generalized Casson fluid model with heat generation and chemical reaction." Results in physics 7 (2017): 789-800. https://doi.org/10.1016/i.rinp.2017.01.025

[34] Parvin, Shahanaz, Siti Suzilliana Putri Mohamed Isa, Norihan Md Arifin, and Fadzilah Md Ali. "The Magnetohydrodynamics Casson Fluid Flow, Heat and Mass Transfer Due to the Presence of Assisting Flow and Buoyancy Ratio Parameters." (2021). https://doi.org/10.37934/cfdl.12.8.6475

[35] Abbas, Z., M. Sheikh, and S. S. Motsa. "Numerical solution of binary chemical reaction on stagnation point flow of Casson fluid over a stretching/shrinking sheet with thermal radiation." Energy 95 (2016): 12-20. https://doi.org/10.1016/i.energy.2015.11.039

[36] Fetecau, Constantin, Dumitru Vieru, and Waqas Ali Azhar. "Natural convection flow of fractional nanofluids over an isothermal vertical plate with thermal radiation." Applied Sciences 7, no. 3 (2017): 247. https://doi.org/10.3390/app7030247

[37] Kataria, Hari R., and Harshad R. Patel. "Radiation and chemical reaction effects on MHD Casson fluid flow past an oscillating vertical plate embedded in porous medium." Alexandria Engineering Journal 55, no. 1 (2016): 583-595. https://doi.org/10.1016/i.aej.2016.01.019

[38] Zakaria, Muhamad Najib, Abid Hussanan, llyas Khan, and Sharidan Shafie. "The effects of radiation on free convection flow with ramped wall temperature in Brinkman type fluid." Jurnal Teknologi 62, no. 3 (2013). https://doi.org/10.11113/it.v62.1886 
[39] Hassanzadeh, Hassan, and Mehran Pooladi-Darvish. "Comparison of different numerical Laplace inversion methods for engineering applications." Applied mathematics and computation 189, no. 2 (2007): 1966-1981. https://doi.org/10.1016/i.amc.2006.12.072

[40] Saqib, Muhammad, Ilyas Khan, and Sharidan Shafie. "Application of fractional differential equations to heat transfer in hybrid nanofluid: modeling and solution via integral transforms." Advances in Difference Equations 2019, no. 1 (2019): 1-18. https://doi.org/10.1186/s13662-019-1988-5 